\title{
Elementary Landscape Decomposition of the Hamiltonian Path Optimization Problem
}

\author{
Darrell Whitley ${ }^{1}$ and Francisco Chicano ${ }^{2}$ \\ 1 Dept. of Computer Science, Colorado State University, Fort Collins CO, USA * \\ whitley@cs.colostate.edu

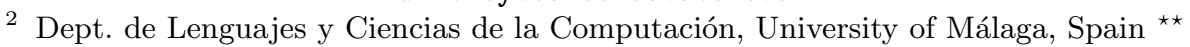 \\ chicano@lcc.uma.es
}

\begin{abstract}
There exist local search landscapes where the evaluation function is an eigenfunction of the graph Laplacian that corresponds to the neighborhood structure of the search space. Problems that display this structure are called "Elementary Landscapes" and they have a number of special mathematical properties. The problems that are not elementary landscapes can be decomposed in a sum of elementary ones. This sum is called the elementary landscape decomposition of the problem. In this paper, we provide the elementary landscape decomposition for the Hamiltonian Path Optimization Problem under two different neighborhoods.
\end{abstract}

Keywords: Landscape theory, elementary landscapes, hamiltonian path optimization, quadratic assignment problem

\section{Introduction}

Grover [7] originally observed that there exist neighborhoods for Traveling Salesperson Problem (TSP), Graph Coloring, Min-Cut Graph Partitioning, Weight Partitioning, as well as Not-All-Equal-SAT that can be modeled using a wave equation borrowed from mathematical physics. Stadler named this class of problems "elementary landscapes" and showed that if a landscape is elementary, the objective function is an eigenfunction of the Laplacian matrix that describes the connectivity of the neighborhood graph representing the search space. When the

\footnotetext{
* This research was sponsored by the Air Force Office of Scientific Research, Air Force Materiel Command, USAF, under grant number FA9550-11-1-0088. The U.S. Government is authorized to reproduce and distribute reprints for Governmental purposes notwithstanding any copyright notation thereon.

** It was also partially funded by the Fulbright program, the Spanish Ministry of Education ("José Castillejo" mobility program), the University of Málaga (Andalucía Tech), the Spanish Ministry of Science and Innovation and FEDER under contract TIN2011-28194 and VSB-Technical University of Ostrava under contract OTRI 8.06/5.47.4142. The authors would also like to thank the organizers and participants of the seminar on Theory of Evolutionary Algorithms (13271) at Schloß Dagstuhl Leibniz-Zentrum für Informatik.
} 
landscape is not elementary it is always possible to write the objective function as a sum of elementary components, called the elementary landscape decomposition (ELD) of a problem [6].

Landscape theory has been proven to be quite effective computing summary statistics of the optimization problem. Sutton et al. [14] show how to compute statistical moments over spheres and balls of arbitrary radius around a given solution in polynomial time using the ELD of pseudo-Boolean functions. Chicano and Alba [4] and Sutton and Whitley [13] have shown how the expected value of the fitness of a mutated individual can be exactly computed using the ELD. Measures like the autocorrelation length and the autocorrelation coefficient can be efficiently computed using the ELD of a problem. Chicano and Alba [5] proved that Fitness-Distance Correlation can be exactly computed using landscape theory for pseudo-Boolean functions with one global optimum.

The landscape analysis of combinatorial optimization problems has also inspired the design of new and more efficient search methods. This is the case of the average-constant steepest descent operator for NK-landscapes and MAX-kSAT of Whitley et al. [15], the second order partial derivatives of Chen et al. [3] and the hyperplane initialization for MAX-kSAT of Hains et al. [8].

However, the first step in the landscape analysis is to find the ELD of the problem. In this paper, we present the ELD of the Hamiltonian Path Optimization Problem (HPO) for two different neighborhoods: the reversals and the swaps. This problem has applications in DNA fragment assembling and the construction of radiation hybrid maps. The remainder of the paper is organized as follows. In Section 2 we present a short introduction to landscape theory. Section 3 presents the HPO and its relationship with QAP. In Sections 4 and 5 we present the landscape structre of HPO for the reversals and swaps neighborhood, respectively. Finally, Section 6 concludes the paper and outlines future directions.

\section{Background on Landscape Theory}

Let $(X, N, f)$ be a landscape, where $X$ is a finite set of candidate solutions, $f: X \rightarrow \mathbb{R}$ is a real-valued function defined on $X$ and $N: X \rightarrow 2^{X}$ is the neighborhood operator. The pair $(X, N)$ is called configuration space and induces a graph in which $X$ is the set of nodes and an arc between $(x, y)$ exists if $y \in N(x)$. The adjacency and degree matrices of the neighborhood $N$ are:

$$
\mathbf{A}_{x, y}=\left\{\begin{array}{l}
1 \text { if } y \in N(x), \\
0 \text { otherwise }
\end{array} \quad \mathbf{D}_{x, y}= \begin{cases}|N(x)| & \text { if } x=y, \\
0 & \text { otherwise }\end{cases}\right.
$$

We restrict our attention to regular neighborhoods, where $|N(x)|=d>0$ for a constant $d$, for all $x \in X$. Then, the degree matrix is $\mathbf{D}=d \mathbf{I}$, where $\mathbf{I}$ is the identity matrix. The Laplacian matrix $\boldsymbol{\Delta}$ associated to the neighborhood is defined by $\boldsymbol{\Delta}=\mathbf{A}-\mathbf{D}$. In the case of regular neighborhoods it is $\boldsymbol{\Delta}=\mathbf{A}-d \mathbf{I}$. Any discrete function, $f$, defined over the set of candidate solutions can be characterized as a vector in $\mathbb{R}^{|X|}$. Any $|X| \times|X|$ matrix can be interpreted as a 
linear map that acts on vectors in $\mathbb{R}^{|X|}$. For example, the adjacency matrix $\mathbf{A}$ acts on function $f$ as follows $(\mathbf{A} f)(x)=\sum_{y \in N(x)} f(y)$, where the component $x$ of $(\mathbf{A} f)$ is the sum of the function values of all the neighbors of $x$. Stadler defines the class of elementary landscapes where the function $f$ is an eigenvector (or eigenfunction) of the Laplacian up to an additive constant [11].

Definition 1. Let $(X, N, f)$ be a landscape and $\boldsymbol{\Delta}$ the Laplacian matrix of the configuration space. The landscape is said to be elementary if there exists a constant $b$, which we call offset, and an eigenvalue $\lambda$ of $-\boldsymbol{\Delta}$ such that $(-\boldsymbol{\Delta})(f-b)=\lambda(f-b)$. When the neighborhood is clear from the context we also say that $f$ is elementary.

We use $-\boldsymbol{\Delta}$ instead of $\boldsymbol{\Delta}$ in the definition to avoid negative eigenvalues, since $\boldsymbol{\Delta}$ is negative semidefinite. In connected neighborhoods, where the graph related to the configuration space $(X, N)$ is connected, the offset $b$ is the average value of the function over the whole search space: $b=\bar{f}$. Taking into account basic results of linear algebra, it can be proven that if $f$ is elementary with eigenvalue $\lambda, a f+b$ is also elementary with the same eigenvalue $\lambda$. If $f$ is an elementary function with eigenvalue $\lambda$, then the average in the neighborhood of a solution can computed as:

$$
\underset{y \in N(x)}{\operatorname{Avg}(f(y))}=f(x)+\frac{\lambda}{d}(\bar{f}-f(x))
$$

known as Grover's wave equation [7], which claims that the average fitness in the neighborhood of a solution $x$ can be computed from the fitness of $x$. The reader interested in Landscape Theory can refer to the survey by Reidys and Stadler [11].

\section{Hamiltonian Path Optimization Problem}

Given a complete edge-weighted graph, the Hamiltonian Path Optimization Problem (HPO) consists in finding the minimum-cost path that visits all vertices in the graph exactly once, without visiting any vertex twice. This problem has applications in DNA "Linkage Marker" ordering, and in manufacturing, specifically in "set-up cost" minimization. It is similar to the Traveling Salesperson Problem (TSP), except that the result is not a circuit. The path can start at any vertex in the graph and it can end at any other vertex. There exists a polynomial time tranformation that converts any Hamiltonian Path Optimization Problem over $n$ vertices into a Traveling Salesman Problem over $n+1$ vertices. Assuming the goal is minimization and that all of the edge weights are positive, an additional dummy vertex can be added that is connected to every other vertex with zero cost. The optimal Hamiltonian Path is thus transformed into a circuit by using the dummy vertex (and two zero cost edges) to return to the beginning of the path: the Hamiltonian Path and the Circuit have exactly the same cost. We will return to this observation in the conclusions. 
The solutions for the HPO problem can be modeled as permutations, which indicate in which order all the vertices are visited. Let us number all the vertices of the graph and let $w$ be the weight matrix, which we will consider symmetric, where $w_{p, q}$ is the weight of the edge $(p, q)$. The fitness function for HPO is:

$$
f(\pi)=\sum_{i=1}^{n-1} w_{\pi(i), \pi(i+1)}
$$

where $\pi$ is a permutation and $\pi(i)$ is the $i$-th element in that permutation. HPO is a subtype of a more general problem: the Quadratic Assignment Problem (QAP) [2]. Given two matrices $r$ and $w$ the fitness function of QAP is:

$$
f_{Q A P}(\pi)=\sum_{i, j=1}^{n} r_{i, j} w_{\pi(i), \pi(j)}
$$

We can observe that (3) generalizes (2) if $w$ is the weight matrix for HPO and we define $r_{i, j}=\delta_{i+1}^{j}$, where $\delta$ is the Kronecker delta. HPO has applications in Bioinformatics, in particular in DNA fragment assembling [10] and the construction of radiation hybrid maps [1].

\section{Landscape for Reversals}

Given a permutation $\pi$ and two positions $i$ and $j$ with $1 \leq i<j \leq n$, we can form a new permutation $\pi^{\prime}$ by reversing the elements between $i$ and $j$ (inclusive). Formally, the new permutation is defined as:

$$
\pi^{\prime}(k)= \begin{cases}\pi(k) & \text { if } k<i \text { or } k>j \\ \pi(j+i-k) & \text { if } i \leq k \leq j\end{cases}
$$

Figure 1(a) illustrates the concept of reversal. The reversal neighborhood $N_{R}(\pi)$ of a permutation $\pi$ contains all the permutations that can be formed by applying reversals to $\pi$. Each reversal can be identified by a pair $[i, j]$, which are the starting and ending positions of the reversal. We use square brackets in the reversals to distinguish them from swaps. Then, we have $\left|N_{R}(\pi)\right|=n(n-1) / 2$.

In the context of HPO, where we consider a symmetric cost matrix, the solution we obtain after applying the reversal $[i, j]=[1, n]$ to $\pi$ has the same objective value as $\pi$, since it is simply a reversal of the complete permutation. For this reason, we will remove this reversal from the neighborhood. We will call the new neighborhood the reduced reversal neighborhood, denoted with $N_{R R}$ to distinguish it from the original reversal neighborhood. We have $\left|N_{R R}(\pi)\right|=$ $n(n-1) / 2-1$. In the remaining of this section we will refer always to the reduced reversal neighborhood unless otherwise stated. The landscape analyzed in this section is composed of the set of permutations of $n$ elements, the reduced reversal neighborhood and the objective function (2). We will use the component model explained in the next section to prove that this landscape is elementary. 


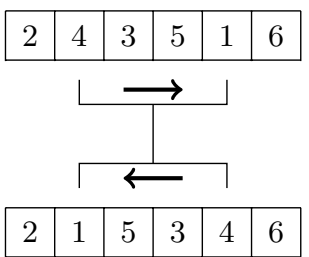

(a) Reversal

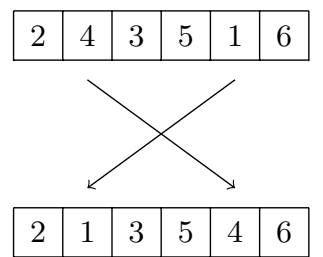

(b) Swap

Fig. 1. Examples of reversal and swap for a permutation of size 6 .

\subsection{Component Model}

Whitley and Sutton developed a "component" based model that makes it easy to identify elementary landscapes [17]. Let $C$ be a set of "components" of a problem. Each component $c \in C$ has a weight (or cost) denoted by $w(c)$. A solution $x \subseteq C$ is a subset of components and the evaluation function $f(x)$ maps each solution $x$ to the sum of the weights of the components in $x: f(x)=\sum_{c \in x} w(c)$. Finally, let $C-x$ denote the subset of components that do not contribute to the evaluation of solution $x$. Note that the sum of the weights of the components in $C-x$ is computed by $\sum_{c \in C} w(c)-f(x)$. In the context of the component model, Grover's wave equation can be expressed as:

$$
\underset{y \in N(x)}{\operatorname{Avg}(f(y))}=f(x)-p_{1} f(x)+p_{2}\left(\sum_{c \in C} w(c)-f(x)\right),
$$

where $p_{1}=\alpha / d$ is the (sampling) rate at which components that contribute to $f(x)$ are removed from solution $x$ to create a neighboring solution $y \in N(x)$, and $p_{2}=\beta / d$ is the rate at which components in the set $C-x$ are sampled to create a neighboring solution $y \in N(x)$. By simple algebra,

$$
\underset{y \in N(x)}{\operatorname{Avg}(f(y)})=f(x)-p_{1} f(x)+p_{2}\left(\sum_{w \in C} w(c)-f(x)\right)=f(x)+\frac{\lambda}{d}(\bar{f}-f(x)),
$$

where $\lambda=\alpha+\beta$, and $\bar{f}=\beta /(\alpha+\beta) \sum_{c \in C} w(c)[16]$.

\subsection{Proof of Elementariness}

Let us start presenting two lemmas.

Lemma 1. Given the $n$ ! possible Hamiltonian Paths over $n$ vertices, all edges appear the same number of times in the $n !$ solutions and this number is $2(n-1)$ !.

Proof. Each edge $(u, v)$ of the graph can be placed in positions $1,2, \ldots, n-1$ of the permutation. This means a total of $n-1$ positions where $(u, v)$ can be placed. For each position it can appear as $u$ followed by $v$ or $v$ followed by $u$. 
Once it is fixed in one position the rest of the positions of the permutation must be filled with the remaining $n-2$ elements. This can be done in $(n-2)$ ! ways. Thus, each edge $(u, v)$ of the graph appears in $2 \cdot(n-1) \cdot(n-2) !=2(n-1)$ ! permutations.

Lemma 2. The average fitness value of the Hamiltonian Path Optimization Problem over the entire solution space is: $\bar{f}=2 / n \sum_{c \in C} w(c)$.

Proof. There are $n(n-1) / 2$ edges in the cost matrix, and there are $n-1$ edges in any particular solution. Since all edges uniformly appear in the set of all possible solutions, Lemma 1 implies:

$$
\bar{f}=\frac{2(n-1) ! \sum_{c \in C} w(c)}{n !}=2 / n \sum_{c \in C} w(c) .
$$

The next theorem presents the main result of this section: it proves that HPO is an elementary landscape for the reduced reversals neighborhood.

Theorem 1. For the Hamiltonian Path Optimization Problem,

$$
\underset{y \in N_{R R}(x)}{\operatorname{Avg}(f(y))}=f(x)+\frac{n}{n(n-1) / 2-1}(\bar{f}-f(x)),
$$

and it is an elementary landscape for the reduced reversals neighborhood.

Proof. First, we need to show that all of the $n-1$ edges that contributes to $f(x)$ are uniformly broken by the reverse operator when all of the neighbors of $f(x)$ are generated. The segments to be reversed range in length from 2 to $n-1$. If the length of the segment is $i$, then the number of possible segments of length $i$ is $n-(i-1)$. Let us consider reversals of length $i$ and $n-i+1$ together, where $1<i \leq n / 2$. The reversal of length $i$ will break the first and last $i-1$ edges in the permutation only once, but it will break all interior edges twice. However the reversal of length $n-i+1$ will only break the first and last $i-1$ edges, and it will break these edges only once. Thus, grouping these together, all edges are broken twice for each value of $i$.

When $n$ is even, the reversals of length $n / 2$ and $n / 2+1$ are grouped together and the pairing is complete. Thus, for $i=2$ to $n / 2$ all edges are broken twice, and thus every edge is broken $2(n / 2-1)=(n-2)$ times and $\alpha=n-2$.

When $n$ is odd, reversals of length $(n+1) / 2$ are a special case. For $i=2$ to $(n-1) / 2$ all edges are broken twice, so all edges have been broken $2((n-1) / 2-$ $1)=(n-3)$ times, but this does not count the reversal of length $(n+1) / 2$. When the reversal of length $(n+1) / 2$ is applied (and is not paired with another reversal) each edge is broken exactly once. Thus all edges are now broken $(n-2)$ times and $\alpha=n-2$.

Next we show that all weights in the cost matrix in the set $C-x$ are uniformly sampled by the reverse operator. Consider the vertex $v_{i}$ in the permutation. Holding $i$ fixed for the moment, consider all cuts that are made at location $i$ 
and all feasible locations $j<i$. When all possible values of $j$ are considered, this causes all of the vertices in the permutation left of vertex $v_{i}$ to come into a position adjacent to $v_{i}$ except for $v_{i-1}$ which is already adjacent. Next consider a cut at location $i+1$ ( $i$ is still fixed) and all feasible locations $m>i+1$. When all of the possible of value of $m$ are considered all of the vertices in the permutation to the right of vertex $v_{i}$ are moved into a position adjacent to $v_{i}$ except $v_{i+1}$. Thus, in these cases $v_{i}$ does not move, but every edge not in the solution $x$ that is incident on vertex $i$ is sampled once. Since this is true for all vertices, it follows that every edge not in the current solution is sampled twice $(\beta=2)$ : once for each of the vertices in which it is incident. Therefore, summing over all the neighbors: $d \cdot \operatorname{Avg}(f(y))_{y \in N(x)}=d \cdot f(x)-(n-2) f(x)+2\left(\sum_{c \in C} w(c)-f(x)\right)$.

Computing the average over the neighborhood and taking into account the result of Lemma 2:

$$
\begin{aligned}
\underset{y \in N(x)}{\operatorname{Avg}(f(y))} & =f(x)-\frac{n-2}{n(n-1) / 2-1} f(x)+\frac{2}{n(n-1) / 2-1}\left(\sum_{c \in C} w(c)-f(x)\right) \\
& =f(x)+\frac{n}{n(n-1) / 2-1}(\bar{f}-f(x)) .
\end{aligned}
$$

\section{$5 \quad$ Landscape Structure for Swaps}

Given a permutation $\pi$, we can build a new permutation $\pi^{\prime}$ by swapping two positions $i$ and $j$ in the permutation. The new permutation is defined as:

$$
\pi^{\prime}(k)=\left\{\begin{array}{l}
\pi(k) \text { if } k \neq i \text { and } k \neq j, \\
\pi(i) \text { if } k=j \\
\pi(j) \text { if } k=i
\end{array}\right.
$$

Figure 1(b) illustrates the concept of swap. The swap neighborhood $N_{S}(\pi)$ of a permutation $\pi$ contains all the permutations that can be formed by applying swaps to $\pi$. Each swap can be identified by the pair $(i, j)$ of positions to swap. The cardinality of the swap neighborhood is $\left|N_{S}(\pi)\right|=n(n-1) / 2$. Unless otherwise stated, we will refer always to the swap neighborhood in this section.

We will analyze the landscape composed of the set of permutations of $n$ elements, the swap neighborhood and the objective function (2). The approach used for this analysis will be different than the one in the previous section. Instead of using the component model we will base our results in the analysis of the QAP landscape done in [6]. The reasons for not using the component model will be discussed at the end of the section.

\subsection{Previous Results for QAP}

According to Chicano et al. [6], the ELD of the QAP for the swap neighborhood is composed of three components. The objective function analyzed in [6] is more general than (3), it corresponds to the Lawler version of QAP [9]: 


$$
f(\pi)=\sum_{i, j, p, q=1}^{n} \psi_{i, j, p, q} \delta_{\pi(i)}^{p} \delta_{\pi(j)}^{q},
$$

where $\delta$ is the Kronecker delta. The correspondence with (3) is $\psi_{i, j, p, q}=r_{i, j} w_{p, q}$. The elementary landscape decomposition of the Lawler QAP is given next.

Theorem 2 (from [6]). The landscape composed of the permutations of $n$ elements, the swap neighborhood and the objective function (6) can be decomposed as the sum of at most three elementary landscapes with eigenvalues $\lambda_{1}=n$, $\lambda_{2}=2 n$ and $\lambda_{3}=2(n-1)$. The definition of these elementary components are:

$$
\begin{aligned}
f_{n}(\pi) & =\frac{1}{n(n-2)} \sum_{\substack{i, j=1 \\
i \neq j}}^{n} \sum_{\substack{p, q=1 \\
p \neq q}}^{n} \psi_{i, j, p, q} \phi_{(i, j),(p, q)}^{n}(\pi)+\sum_{i, p=1}^{n} \psi_{i, i, p, p} \delta_{\pi(i)}^{p}, \\
f_{2 n}(\pi) & =\frac{1}{2 n} \sum_{\substack{i, j=1 \\
i \neq j}}^{n} \sum_{\substack{p, q=1 \\
p \neq q}}^{n} \psi_{i, j, p, q} \phi_{(i, j),(p, q)}^{2 n}(\pi), \\
f_{2(n-1)}(\pi) & =\frac{1}{2(n-2)} \sum_{\substack{i, j=1 \\
i \neq j}}^{n} \sum_{\substack{p, q=1 \\
p \neq q}}^{n} \psi_{i, j, p, q} \phi_{(i, j),(p, q)}^{2(n-1)}(\pi),
\end{aligned}
$$

where the $\phi$ functions are defined using as base the $\phi_{(i, j),(p, q)}^{\alpha, \beta, \gamma, \zeta}$ function as $\phi_{(i, j),(p, q)}^{n}=$ $\phi_{(i, j),(p, q)}^{n-1,3-n, 0,2-n, 1-n}, \phi_{(i, j),(p, q)}^{2 n}=\phi_{(i, j),(p, q)}^{n-1,3-n, 0,2,1}$ and $\phi_{(i, j),(p, q)}^{2(n-1)}=\phi_{(i, j),(p, q)}^{n-3, n-3,0,0,1}$. The $\phi_{(i, j),(p, q)}^{\alpha, \gamma, \gamma, \zeta}$ function is defined as:

$$
\phi_{(i, j),(p, q)}^{\alpha, \beta, \gamma, \zeta}(\pi)=\left\{\begin{array}{l}
\alpha \text { if } \pi(i)=p \wedge \pi(j)=q, \\
\beta \text { if } \pi(i)=q \wedge \pi(j)=p, \\
\gamma \text { if } \pi(i)=p \oplus \pi(j)=q, \\
\varepsilon \text { if } \pi(i)=q \oplus \pi(j)=p, \\
\zeta \text { if } \pi(i) \notin\{p, q\} \wedge \pi(j) \notin\{p, q\},
\end{array}\right.
$$

where $\oplus$ denotes the exclusive OR. The function $f$ can be written in a compact form as: $f=f_{n}+f_{2 n}+f_{2(n-1)}$.

In the next subsection we analyze this decomposition, providing some properties that are useful to find the elementary landscape decomposition of the HPO under the swap neighborhood.

\subsection{Elementary Landscape Decomposition of the HPO}

The functions $\phi_{(i, j),(p, q)}^{n}, \phi_{(i, j),(p, q)}^{2 n}$, and $\phi_{(i, j),(p, q)}^{2(n-1)}$ defined above have some properties that make $f_{2 n}$ and $f_{2(n-1)}$ vanish when the matrices $r$ and $w$ fulfill some concrete conditions. The next proposition summarizes these properties: 
Proposition 1. The functions $\phi_{(i, j),(p, q)}^{n}, \phi_{(i, j),(p, q)}^{2 n}$, and $\phi_{(i, j),(p, q)}^{2(n-1)}$ defined in Theorem 2 hold the next equalities:

$$
\begin{aligned}
& \phi_{(i, j),(p, q)}^{\lambda}=\phi_{(j, i),(q, p)}^{\lambda} \quad \text { for } \lambda=n, 2 n, 2(n-1), \\
& \phi_{(i, j),(p, q)}^{2 n}+\phi_{(j, i),(p, q)}^{2 n}=2, \\
& \phi_{(i, j),(p, q)}^{2(n-1)}=\phi_{(j, i),(p, q)}^{2(n-1)} .
\end{aligned}
$$

Proof. The first equation (11) follows from the fact that exchanging $i$ and $j$ at the same time as $p$ and $q$ in any of the branch conditions of the function (10) leaves the condition unchanged, thus the function is the same.

In order to prove Eqs. (12) and (13) we can observe that exchanging $i$ and $j$ in (10) is equivalent to swapping the values of $\alpha$ and $\beta$ (first and second branches) and those of $\gamma$ and $\varepsilon$ (third and fourth branches). The fifth branch is left unchanged. Thus, Eq. (13) is a direct consequence of this swap of branch values and (12) can be proven as follows:

$$
\begin{aligned}
\phi_{(i, j),(p, q)}^{2 n}(\pi)+ & \phi_{(j, i),(p, q)}^{2 n}(\pi) \\
= & \begin{aligned}
n-1 \\
3-n \\
0 \\
2 \\
1
\end{aligned}+\left\{\begin{array}{rl}
3-n \\
n-1 \\
2 \\
0 \\
1
\end{array}=\left\{\begin{array}{l}
2 \text { if } \pi(i)=p \wedge \pi(j)=q, \\
2 \text { if } \pi(i)=q \wedge \pi(j)=p, \\
2 \text { if } \pi(i)=p \oplus \pi(j)=q, \\
2 \text { if } \pi(i)=q \oplus \pi(j)=p, \\
2 \text { if } \pi(i) \notin\{p, q\} \wedge \pi(j) \notin\{p, q\} .
\end{array}\right.\right.
\end{aligned}
$$

Let us now analyze the consequences of these properties for the elementary landscape decomposition of a QAP instance.

Theorem 3. Let us consider the elementary landscape decomposition of (6) for the swap neighborhood given in Theorem 2. Then we have:

- If any of the matrices $r$ or $w$ is symmetric then $f_{2 n}$ is constant.

- If any of the matrices $r$ or $w$ is antisymmetric then $f_{2(n-1)}$ is zero.

Proof. We will prove the theorem assuming that $r$ has the required property (symmetric or antisymmetric) and then we will prove that the corresponding elementary components are still constant or zero if we exchange $r$ and $w$.

Let us assume that matrix $r$ is symmetric $\left(r_{i, j}=r_{j, i}\right)$, then we can write:

$$
\begin{aligned}
f_{2 n} & =\frac{1}{2 n} \sum_{\substack{i, j=1 \\
i<j}}^{n} \sum_{\substack{p, q=1 \\
p \neq q}}^{n} r_{i, j} w_{p, q}\left(\phi_{(i, j),(p, q)}^{2 n}+\phi_{(j, i),(p, q)}^{2 n}\right) \quad \text { by (12) } \\
& =\frac{1}{2 n} \sum_{\substack{i, j=1 \\
i<j}}^{n} \sum_{\substack{p, q=1 \\
p \neq q}}^{n} 2 r_{i, j} w_{p, q}=\frac{1}{n}\left(\sum_{\substack{i, j=1 \\
i<j}}^{n} r_{i, j}\right)\left(\sum_{\substack{p, q=1 \\
p \neq q}}^{n} w_{p, q}\right),
\end{aligned}
$$


which is a constant value that only depends on the instance, not the solution. Let us now assume that $r$ is antisymmetric $\left(r_{i, j}=-r_{j, i}\right)$, then we can write:

$$
f_{2(n-1)}=\frac{1}{2(n-2)} \sum_{\substack{i, j=1 \\ i<j}}^{n} \sum_{\substack{p, q=1 \\ p \neq q}}^{n} r_{i, j} w_{p, q}\left(\phi_{(i, j),(p, q)}^{2(n-1)}-\phi_{(j, i),(p, q)}^{2(n-1)}\right)=0 .
$$

Let us call $g(\pi)$ to the fitness function we obtain by exchanging matrices $r$ and $w$ in the definition of the fitness function (6) and let us call $f(\pi)$ to the original fitness function. The relationship between these two functions is as follows:

$$
\begin{aligned}
g(\pi) & =\sum_{i, j=1}^{n} \sum_{p, q=1}^{n} w_{i, j} r_{p, q} \delta_{\pi(i)}^{p} \delta_{\pi(j)}^{q} \quad \text { renaming indices }(p \leftrightarrow i \text { and } q \leftrightarrow j) \\
& =\sum_{p, q=1}^{n} \sum_{i, j=1}^{n} w_{p, q} r_{i, j} \delta_{\pi(p)}^{i} \delta_{\pi(q)}^{j}=\sum_{i, j=1}^{n} \sum_{p, q=1}^{n} r_{i, j} w_{p, q} \delta_{\pi^{-1}(i)}^{p} \delta_{\pi^{-1}(j)}^{q}=f\left(\pi^{-1}\right) .
\end{aligned}
$$

The elementary landscape decomposition of $g(\pi)=g_{n}(\pi)+g_{2 n}(\pi)+g_{2(n-1)}(\pi)$ can thus be written based on the elementary landscape decomposition of $f(\pi)$ as: $g_{\lambda}(\pi)=f_{\lambda}\left(\pi^{-1}\right)$ for $\lambda=n, 2 n, 2(n-1)$. If the $w$ matrix of the original fitness function $f$ is symmetric or antisymmetric then the $r$ matrix of the $g$ function will have the same property, and, as we have proven above, $g_{2 n}=$ constant or $g_{2(n-1)}=0$, respectively. But this means that also $f_{2 n}=$ constant or $f_{2(n-1)}=0$, respectively.

Since the addition of constant values does not affect the number of elementary components of a landscape, when one of the components is constant in the previous theorem, this component vanishes from the elementary landscape decomposition. In other words, the number of elementary components is reduced whenever any of the conditions in the previous theorem holds. For example, an instance of QAP having a symmetric $w$ will have at most two elementary components, instead of three. The conditions can co-occur in the same instance. If an instance of QAP has one of the matrices symmetric and the other one antisymmetric it will be an elementary landscape, since $f_{2 n}$ and $f_{2(n-1)}$ are constants. For HPO we have the next result.

Corollary 1. The Hamiltonian Path Optimization Problem with symmetric cost matrix is the sum of at most two elementary components in the swap neighborhood. One possible expression for these two components is:

$$
\begin{aligned}
f_{n} & =\frac{1}{n(n-2)} \sum_{i=1}^{n-1} \sum_{\substack{p, q=1 \\
p \neq q}} w_{p, q} \phi_{(i, i+1),(p, q)}^{n}+\frac{n-1}{n} \sum_{\substack{p, q=1 \\
p<q}}^{n} w_{p, q}, \\
f_{2(n-1)} & =\frac{1}{2(n-2)} \sum_{i=1}^{n-1} \sum_{\substack{p, q=1 \\
p \neq q}} w_{p, q} \phi_{(i, i+1),(p, q)}^{2(n-1)},
\end{aligned}
$$


where $f_{n}$, and $f_{2(n-1)}$ are elementary with eigenvalues $n$ and $2(n-1)$, respectively, and the objective function of HPO $f$ can be written as: $f=f_{n}+f_{2(n-1)}$.

Proof. Since the matrix $w$ is symmetric in the HPO, according to Theorem 3 the $f_{2 n}$ component is constant and its value is: $f_{2 n}=\frac{n-1}{n} \sum_{\substack{p, q=1 \\ p<q}} w_{p, q}$, where we use (14) and take into account that the $r$ matrix contains $n-1$ ones in the upper triangle. We sum this constant to the $f_{n}$ component (without changing its elementariness) and we have the claimed result after some algebraic simplifications in the equations.

The landscape of HPO with the swap neighborhood is the sum of two elementary landscapes. This is the main reason why the component model is not useful for this analysis. The component model can only be used to prove that a landscape is elementary but it is not able to describe landscapes that are sums of more than one elementary component. Furthermore, it is not able to prove the elementariness of some elementary landscapes. This happens, in particular, in elementary landscapes with irrational eigenvalues.

Another mathematical tool to provide the elementary landscape decomposition of optimization problems is the spectral theory of quasi-Abelian Cayley graphs. When the solution set is an Abelian group this theory provides an easy and elegant elementary landscape decomposition of any objective function. This happens for example, in the case of the $q$-ary hypercube [12]. When the solution set is not an Abelian group, as in the case of the permutations, the math involved is more complex but still give elegant answers if the set of generators that define the neighborhood is the union of conjugacy classes of the group [11]. The generators of the swap neighborhood (2-cycles) form a conjugacy class, so the spectral theory could be applied in this case; but the generators of the reversal (and reduced reversal) neighborhood are not the union of conjugacy classes, what makes the component model to be, as far as we know, the best methodology to prove the elementariness of the landscape.

\section{Conclusions and Future Work}

In this work we have proven that the Hamiltonian Path Optimization Problem is elementary under the reversals neighborhood and is the sum of two elementary components in the case of the swaps neighborhood. We have also provided the expressions for the elementary components in the latter case.

In the two neighborhoods considered in the paper the number of moves is $\Theta\left(n^{2}\right)$. For problems that are extremely large, e.g., with billions of vertices, it may not be reasonable to look at all possible moves. On the other hand, some form of heuristic might be used to decide if there exists a smaller subset of locations where the permutation might be reasonably be broken, while other edges are not broken. In this way, a problem with billions of vertices might be mapped onto a problem with only hundreds of thousands vertices. This leads to the so-called Partial Neighborhoods [17]. An interesting question to analyze is the landscape structure for these partial neighborhoods. 
At the beginning of Section 3, we noted that every Hamiltonian Path Optimization Problem over $n$ vertices can be converted into a Traveling Salesman Problems over $n+1$ vertices. In practice, this means that existing TSP solvers can be use to solve the Hamiltonian Path Optimization Problem. One interesting question is whether the elementary landscapes which exist for the TSP can be mapped onto the elementary landscapes which we have defined for the Hamiltonian Path Optimization Problem.

\section{References}

1. Agarwala, R., Applegate, D.L., Maglott, D., Schuler, G.D.: A fast and scalable radiation hybrid map construction and integration strategy. Genome Research 10(3), 350-364 (2000)

2. Burkard, R.E.: Handbook of Combinatorial Optimization, chap. Quadratic Assignment Problems, pp. 2741-2815. Springer, 2nd edn. (2013)

3. Chen, W., Whitley, D., Hains, D., Howe, A.: Second order partial derivatives for NK-landscapes. In: Proceeding of GECCO. pp. 503-510. ACM (2013)

4. Chicano, F., Alba, E.: Exact computation of the expectation curves of the bit-flip mutation using landscapes theory. In: Proc. of GECCO. pp. 2027-2034 (2011)

5. Chicano, F., Alba, E.: Exact computation of the fitness-distance correlation for pseudoboolean functions with one global optimum. In: Proceedings of EvoCOP. LNCS, vol. 7245, pp. 111-123. Springer (2012)

6. Chicano, F., Whitley, L.D., Alba, E.: A methodology to find the elementary landscape decomposition of combinatorial optimization problems. Evolutionary Computation 19(4), 597-637 (2011)

7. Grover, L.K.: Local search and the local structure of NP-complete problems. Operations Research Letters 12, 235-243 (1992)

8. Hains, D., Whitley, D., Howe, A., Chen, W.: Hyperplane initialized local search for MAXSAT. In: Proceeding of GECCO. pp. 805-812. ACM (2013)

9. Lawler, E.L.: The quadratic assignment problem. Manage. Sci. 9, 586-599 (1963)

10. Parsons, R., Forrest, S., Burks, C.: Genetic algorithms, operators, and DNA fragment assembly. Machine Learning 21, 11-33 (1995)

11. Reidys, C.M., Stadler, P.F.: Combinatorial landscapes. SIAM Review 44(1), 3-54 (2002)

12. Sutton, A.M., Chicano, F., Whitley, L.D.: Fitness function distributions over generalized search neighborhoods in the q-ary hypercube. Evol. Comput. 21(4) (2013)

13. Sutton, A.M., Whitley, D., Howe, A.E.: Mutation rates of the (1+1)-EA on pseudoboolean functions of bounded epistasis. In: Proc. of GECCO. pp. 973-980 (2011)

14. Sutton, A.M., Whitley, L.D., Howe, A.E.: Computing the moments of k-bounded pseudo-boolean functions over hamming spheres of arbitrary radius in polynomial time. Theoretical Computer Science 425, 58-74 (2011)

15. Whitley, D., Chen, W.: Constant time steepest descent local search with lookahead for NK-landscapes and MAX-kSAT. In: Proc. of GECCO. pp. 1357-1364 (2012)

16. Whitley, D., Sutton, A.M., Howe, A.E.: Understanding elementary landscapes. In: Proc. of GECCO. pp. 585-592 (2008)

17. Whitley, L.D., Sutton, A.M.: Partial neighborhoods of elementary landscapes. In: Proc. of GECCO. pp. 381-388 (2009) 